phenotype had better survival to chemotherapy treatment. At the molecular level, we found out that the mitogenactivated protein kinase, phosphoinosytol-3 phosphate/AKT pathways and ABL1 kinase are involved in nuclear F-actin polymerization. Further investigation revealed that ABL1 may phosphorylate Wiskott-Aldrich syndrome protein to activate the key actin nucleation factor Arp2/3 complex to promote nuclear F-actin polymerization in ovarian cancer cells treated with chemotherapy drugs carboplatin or cisplatin. Our preliminary data suggests a potential target in combination with chemotherapy for ovarian cancer treatment, and this necessitates further evaluation by recruiting preclinical models in future studies.

Conclusion: Here, we reveal that ovarian cancer cells with nuclear F-actin undergo less DNA damage under platinum treatment, suggesting that nuclear F-actin may mediate chemotherapy resistance in the context of ovarian cancer. Moreover, we unravel the underlying kinase pathway involved in nuclear F-actin polymerization in ovarian cancer cells, potentially paving the way for a novel strategy of targeting potential kinases in combination with well-established chemotherapy regimens in order to circumvent chemotherapy resistance.

Poster (020)

Imaging in Gynecologic Cancer

https://doi.org/10.3802/jgo.2021.32.\$1.020

\section{The diagnostics of epithelial ovarian cancer: focus on apparent diffusion coefficient map on magnetic resonance imaging using machine learning}

\author{
Heekyoung Song, ' Jae Yeon Woo,' Seongeun Bak, ${ }^{1}$ \\ Imhyeon Kim, ${ }^{1}$ Youn Jin Choi, ${ }^{1}$ Sung Eun Rha, ${ }^{2}$ Seo Yeon Youn, ${ }^{2}$ \\ Shin Ah Oh, ${ }^{3}$ Sung Jong Lee ${ }^{1, *}$ \\ 'Department of Obstetrics and Gynecology, Seoul St. Mary's Hospital, \\ College of Medicine, The Catholic University of Korea, Seoul, Korea \\ (orlando@catholic.ac.kr) \\ 2Department of Radiology, Seoul St. Mary's Hospital, College of Medicine, The \\ Catholic University of Korea, Seoul, Korea 3NAVER Clova AI, Seongnam, Korea
}

Objective: To compare radiologic features including mean apparent diffusion coefficient (ADC) value of uterus magnetic resonance imaging (MRI) among the histology types of epithelial ovarian cancer (EOC).

Methods: This retrospective single center study included patients underwent preoperative uterus MRI and pathologically diagnosed as EOC. On MRI, the proper image of the axial ADC map that contained the largest area of the solid portion was selected, and the region of interest (ROI) of the solid portion were manually drawn by a genecologist and a radiologist with consensus. The mean $\mathrm{ADC}$ value (ADCmean) of solid portion was derived from ROI. The ADCmean in each histology was compared using MannWhitney $\mathrm{U}$ test. The 3 kinds of machine learning (ML) algorithms also used to evaluate several values included ADCmean (Random Forest, Gradient Boosting Machine, and XGBoost).

Results: Two hundred patients were finally included with 97 type I patients (low-grade serous carcinoma, $\mathrm{n}=8$; mucinous carcinoma $[\mathrm{MC}], \mathrm{n}=33$; endometroid carcinoma [EC], $\mathrm{n}=21$; clear cell carcinoma [CCC], $\mathrm{n}=35$ ) and 103 type II patients (high-grade serous carcinoma [HGSC], $\mathrm{n}=103$ ). The ADCmean significantly differ between type I and II EOC $(\mathrm{p}<0.001)$. In detail, median ADCmean of HGSC was $1.06 \times 10^{-3} \mathrm{~s} / \mathrm{m}^{2}$ the lowest among all types, and significantly distinguished from MC, EC, and CCC ( $\mathrm{p} \leq 0.001)$. Among 3 types of ML, Gradient Boosting Machine noted the highest accuracy to distinguish type I/II EOC (accuracy: 0.91), XGBoost conducted the highest value in 5 histology of EOC (accuracy: 0.68).

Conclusion: The ADCmean is useful tool to evaluate the histologic type of EOC in preoperative MRI.

Poster (O21)

Epithelial Ovarian Cancer including Borderline Tumor

https://doi.org/10.3802/jgo.2021.32.S1.021

\section{Toxicity profile of olaparib maintenance monotherapy for Japanese platinum-sensitive relapsed ovarian, fallopian tube and primary peritoneal cancer patients}

\section{Tomoko Yoshihama, Tatsuyuki Chiyoda, "Yuka Kuroda, Yusuke Kobayashi, Wataru Yamagami, Daisuke Aoki}

Department of Obstetrics and Gynecology, Keio University School of Medicine, Tokyo, Japan (chiyoda@keio.jp)

Objective: This study aims to investigate the toxicity profile of olaparib maintenance therapy for Japanese population.

Methods: Fifty-two Japanese patients with platinum-sensitive relapsed ovarian, fallopian tube and primary peritoneal cancer who started olaparib maintenance monotherapy following platinum-based chemotherapy from May 2018 to June 2021 at our hospital were included. The information about the adverse effect were collected retrospectively from the medical records. This study was approved by the ethics committee of Keio University, School of Medicine.

Results: The median age was 58 years old (33-80 years), and the median progression-free survival was 17.1 months $(95 \%$ confidence interval=10.6-23.5). The number of previous platinum-based regimens was 2 in 35 patients (67.3\%), 3 in 8 patients $(15.4 \%)$ and $\geq 4$ in 9 patients $(17.3 \%)$. Twenty-nine 
patients $(55.8 \%)$ had presented anemia of grade $1-2$ and 18 patients (34.6\%) had experienced anemia of grade $3-4$, which seemed higher than the previous reports. Five patients received blood transfusions. As for non-hematological toxicity, nausea (25 patients, $48.1 \%$ ), fatigue (20 patients, 38.4\%) and blood creatinine increased (27 patients, $51.9 \%$ ) were observed frequently (any grade). The incidence of blood creatinine increased also seemed higher than the previous studies. One patient discontinued olaparib due to prolonged renal dysfunction. In total, 22 patients (42.3\%) required dose interruption, and 11 patients $(21.2 \%)$ required dose reduction. No patient had suffered from acute myeloid leukemia or myelodysplastic syndromes.

Conclusion: Olaparib maintenance monotherapy was tolerable also for Japanese population, although the toxicity profile might be a bit different from the previous reports (SOLO2, Study 19).

Poster (022)

Epithelial Ovarian Cancer including Borderline Tumor

https://doi.org/10.3802/jgo.2021.32.S1.022

\section{TP53 sequence analysis revealed distinct characteristics of p53 signatures in RRSO samples}

Kenta Masuda," Tomoko Akahane, Yusuke Kobayashi, Tatsuyuki Chiyoda, Kouji Banno, Daisuke Aoki

Keio University School of Medicine, Tokyo, Japan (ma-su-ken.a2@keio.jp)

Objective: Risk-reducing salpingo-oophorectomy (RRSO) is recommended for women with $B R C A 1 / 2$ pathogenic variants upon completion of childbearing. Precursor lesions could be identified in the fallopian tubes of RRSO samples. Serous tubal intraepithelial carcinoma (STIC) is considered a precursor of high-grade serous carcinoma (HGSC), however, the significance of the p53 signature remains unclear. In this study, we analyzed the potency of the p53 signature as a precancerous lesion. Methods: We analyzed the clinicopathological findings and conducted DNA sequencing for TP53 variants of $\mathrm{p} 53$ signatures isolated using laser capture microdissection in 13 patients with $B R C A 1 / 2$ pathogenic variants who underwent RRSO and 17 control patients with the benign gynecologic disease.

Results: p53 signatures were most frequently observed in fimbriae of fallopian tubes from RRSO samples. Between the RRSO group and the control group, there was no significant difference in the percentage of $\mathrm{p} 53$ signatures in fimbriae $(\mathrm{p}=0.17)$, whereas the percentage of pathogenic variants in TP53 variants identified in $\mathrm{p} 53$ signature lesions were significantly higher in the RRSO group than the control $(\mathrm{p}<0.001)$. Conclusion: The characteristics of the $\mathrm{p} 53$ signature may be different between specimens obtained during RRSO and those from controls. The sequence analysis for TP53 revealed distinct characteristics of $\mathrm{p} 53$ signatures as precancerous lesions.

Poster (023)

Non-epithelial Ovarian Cancer

https://doi.org/10.3802/jgo.2021.32.S1.023

\section{Tumor recurrence and fertility in young women with malignant ovarian germ cell tumors who received fertility-sparing surgery}

\section{Seok Mo Kim, " U Chul Ju, Woo Dae Kang}

Department of Obstetrics and Gynecology, Chonnam National University Medical School, Gwangju, Korea (ddiamo94@gmail.com)

Objective: We evaluated the tumor recurrence and fertility outcomes in young women with malignant ovarian germ cell tumors (MOGCTs) who received fertility-sparing surgery (FSS). Methods: We reviewed the medical records of women aged $\leq 40$ years with MOGCTs who received FSS between July 2002 and December 2018, retrospectively. FSS was defined as the preservation of the uterus and at least one adnexa.

Results: Forty-four women were included in the study. The median age of the patients was 22 years (range, 7-39 years). Twenty-nine patients $(65.9 \%)$ received postoperative chemotherapy with bleomycin, etoposide, and cisplatin. During a median follow-up period of 62 months (range, 7-185), 4 patients $(9.1 \%)$ had a recurrence. Of these, 2 patients with dysgerminoma had recurrences at para-aortic lymph nodes and 2 patients with immature teratomas had recurrences at the remaining ovary. Of a total of 44 patients, $37(88.1 \%)$ had regular menstruations. Of 14 women desiring a pregnancy, 12 achieved the term delivery of 12 singleton pregnancies. The pregnancy and live birth rates were $85.7 \%$ and $100 \%$, respectively.

Conclusion: FSS with or without adjuvant chemotherapy is an appropriate option for young women with MOGCTs who wish to preserve their fertility.

Poster (024)

Non-Epithelial Ovarian Cancer

https://doi.org/10.3802/jgo.2021.32.S1.024

\section{Giant ovarian fibrosarcoma-a rare case report}

Pratibha Kumari," Satya Kumari, Sangeeta Pankaj

IGIMS, Patna, India (Pratibhabseb@gmail.com)

Objective: Ovarian fibrosarcoma is very rare malignant neoplasm, accounts for less than $1 \%$ of all ovarian 